Cuestiones de filosofía

ISSN: 0123-5095

Vol. 1 - No. 17

Año 2015

pp. $65-75$

\title{
ACTUALIDAD DE LA SEMIÓTICA COMO GENERADOR HEURÍSTICO EN FILOSOFÍA DE LAS CIENCIAS
}

\author{
Actuality of semiotics as heuristic generator \\ in the philosophy of science
}

José Amílcar Rizzo-Sierra

Universidad Industrial de Santander (Colombia)

Jose.rizzo@colnetwork.com

Ángel José Chacón-Velasco

Universidad Pedagógica y Tecnológica de Colombia

Angel.chacon@uptc.edu.co

Fecha de recepción: 16/11/ 2014

Fecha de evaluación: 14/02/2015

Fecha de aprobación: 1/09/2015

\section{Resumen}

Se propone que la semiótica estructural, gracias a sus profundas raíces filosóficas, podría ser empleada como herramienta de trabajo de naturaleza heurística en filosofía de la ciencia; sin embargo, se anota que esta posibilidad ha sido poco explorada en ambientes académicos, llamados a tomarla en cuenta, al menos partiendo desde una perspectiva intelectual explícita y plenamente consciente; esto es una lástima, puesto que estudios con claro y explícito fundamento semiótico en temáticas propias de la filosofía de la ciencia escasean, mientras que estudios con claro trasfondo semiótico, si bien inexplícito y muy seguramente inconsciente para sus autores, permean la discusión de principio en epistemología científica hasta el punto que resulta posible afirmar que algunos de ellos forman parte de las referencias clásicas 
para los estudiosos en la materia sin ser reconocidos como tales. Ejemplos de ambos tipos de trabajos son referenciados y brevemente discutidos.

Palabras clave: Filosofía de la ciencia, Epistemología científica, Empirismo, Semiótica.

\begin{abstract}
It is proposed that thanks to its profound philosophical roots, the science of semiotics could be employed as a tool, heuristic in nature, in philosophy of science. Nevertheless, it is remarked that this possibility has been only slightly explored within relevant academic spheres, at least from a fully conscious and explicit intellectual perspective. This is a pity, since studies with clear and explicit semiotic foundations, within properly subjects on philosophy of science are scarce, while clearly and explicitly semiotic founded studies, tough non-explicit and perhaps even unconscious for their authors, permeate the fundamental intellectual discussion on scientific epistemology, to the point that it seems possible to state that some of them are part of the classical references for the scholars on the matter, without being recognized as such. Both types of work examples are referenced and briefly discussed.
\end{abstract}

Keywords: Philosophy of Science, Semiotics, Scientific Epistemology, Empiricism, Experience.

\title{
INTRODUCCIÓN
}

En términos generales, la semiótica estructural podría tener relevancia en filosofía de la ciencia, puesto que su concepto generatriz es el mismo que el de la epistemología científica, i. e., el de experiencia (Rizzo, 2004, p. 181-182). ¿Por qué?, porque un sistema semiótico se estudia, por principio, como algo cuya estructura proviene de la codificación de un dominio particular de nuestra experiencia; por ejemplo, una lengua natural, quizá el tipo de sistema semiótico más importante estudiado hasta el momento, "puede considerarse como un sistema holístico: por el hecho de estar estructurada de un modo determinado implica una visión del mundo" (Eco, 1994, p. 30). En estos términos, debe repararse claramente en que la estructura de una lengua natural constituye entonces una codificación a través del tiempo de todo aquello que, con sentido, la cultura que la ha desarrollado se encuentra en capacidad 
de interpretar como experiencia. Por esta razón, una lengua natural puede pretender ser "omniefable, es decir, capaz de dar cuenta de toda nuestra experiencia, física y mental, y capaz, pues, de poder expresar sensaciones, percepciones, abstracciones, hasta llegar a la pregunta de por qué existe el ser y no la nada” (Eco, p. 31).

Considerando lo anterior, resulta posible, entonces, proponer que la naturaleza de la semiótica, en relación con sus objetos de estudio, coincide con la-en opinión de innumerables reputados científicos con inquietudes filosóficas-naturaleza fundamental de la ciencia en relación con sus propios objetos de estudio; ello en razón de que tanto semiótica como ciencia reconocen como su fundamento la experiencia, adquirida mediante codificación de funciones semióticas, en el primer caso, y mediante la práctica de cuidadosos experimentos, en el segundo. Semiótica y ciencia se relacionan con sus objetos de estudio mediante el mismo concepto, el de experiencia. Se desarrolla/estudia semiótica estudiando cómo los sistemas semióticos asimilan experiencia al codificarla; se desarrolla/estudia ciencia estudiando cómo se asimila experiencia al interpretarla coherentemente en teorías físicas a partir procedimientos experimentales o experimentos. Ahora bien, para ilustrar esta situación no haría falta mucho trabajo de revisión en la literatura científica. Puede citarse un único, pero representativo, ejemplo con la ayuda del extinto Richard Feynmann, mundialmente conocido por su brillante carrera teórica y pedagógica, cuando nos dice que "The principle of science, the definition, almost, is the following: The test of all knowledge is experiment. Experiment is the sole judge of scientific "truth" (1963, p. 1). Así, desde una perspectiva filosófica, la semiótica se percibe a sí misma en términos de experiencia codificada, mientras que la ciencia se percibe a sí misma en términos de experiencia coherentemente interpretada a partir de experimentos. Para el caso de la ciencia, la filosofía empirista descansa sobre este último principio, y, de paso, su epistemología. De hecho, actualmente sería difícil pensar en otra cosa diferente a una epistemología empirista cuando se hace referencia a una epistemología científica. De manera que la diferencia entre experiencia en semiótica y experiencia en ciencias naturales puede considerarse un tema de aproximación metodológica al mismo fundamento filosófico de las dos disciplinas; ambas son, por supuesto, experiencias, solo que para la epistemología empirista se accede a experiencia mediante experimentos/procedimientos experimentales, mientras intencional y simultáneamente se intentan controlar las condiciones en las que esta se genera. En contextos no científicos, como el semiótico, esta característica metodológica puede darse o no, pero de hacerlo, no es, en general, una iniciativa intencional del sujeto. Por lo anterior, el alegato de considerar semiótica y ciencia con el mismo fundamento filosófico puede estimarse razonablemente justificado. 
Sin embargo, a pesar de esta patente concordancia entre los fundamentos filosóficos de la semiótica y los de la epistemología científica, los autores de estas líneas desconocen la existencia de estudios acerca del concepto de experiencia en contextos de filosofía de la ciencia explícitamente apoyados en herramientas semióticas. Sí, empero, han podido distinguir numerosos ejemplos de estudios en contextos de filosofía de la ciencia que hacen uso inexplícito y muy seguramente inconsciente de herramientas semióticas para estudiar conceptos como el de experiencia. Uso inexplícito y muy seguramente inconsciente quiere decir que estos estudios utilizan, sin referencia o reconocimiento explícito alguno, conceptos y contextos que han sido formalizados en el marco de referencia filosófico de la semiótica estructural. Debe anotarse que desarrollar esta apreciación en detalle, en otros términos, desarrollar detalladamente la posición de que al menos parte de los estudios emprendidos en filosofía de la ciencia son de naturaleza semiótica, no puede hacer parte de este artículo, puesto que lo apartaría de su enfoque inicial, que es el de destacar genéricamente la potencial utilidad de la semiótica estructural en tanto herramienta heurística en filosofía de la ciencia. Sin embargo, es posible aseverar que la posición podría ser desarrollada y argumentada exitosamente, ya que gran parte de la obra de autores como Lakatos, Feyerabend y Popper exhibe características ostensiblemente semióticas.

\section{A PROPÓSITO DE “GENERADOR HEURÍSTICo" EN TORNO A LA CONSTRUCCIÓN DE PERSPECTIVAS EPISTEMOLÓGICAS}

Este artículo se preparó con un enfoque que no pretende desarrollar en detalle la base racional de la posibilidad de que la semiótica estructural pueda tener el rol de generador heurístico en torno a la construcción de perspectivas epistemológicas en filosofía de la ciencia; tal iniciativa escaparía el alcance planteado, puesto que este se centra escuetamente en proponer dicha posibilidad y, a continuación, referir y discutir brevemente un par de casos en la epistemología científica clásica donde esta ha podido haberse consumado ya. Ahora bien, es claro que un comentario acerca de qué podría significar este rol no estaría de más.

En términos generales, en filosofía el término heurística hace referencia a cuando pragmáticamente se echa mano de algo con el fin de satisfacer algunos propósitos referentes a otro algo, como pueden serlo la resolución de problemas, el avance epistemológico y la exploración filosófica. También se habla de proponer y emplear artefactos -generadores heurísticos-, en este sentido. Esas herramientas de las que se echa mano, esos artefactos, pueden ser de muchas clases; cualquier entidad que 
se muestre útil en la comprensión o el avance epistemológico sobre otra entidad diferente a ella misma puede recibir el mote de herramienta, artefacto o generador heurístico. Entre algunos ejemplos de artefactos heurísticos se encuentran los modelos teóricos y el uso de herramientas figurativas en discusiones formales. Más allá de eso, todo tipo de convención, procedimiento o metodología (laxa o no) que permita que una entidad pueda comprender o acceder epistemológicamente a otra puede considerarse también algún tipo de heurística.

En el contexto que nos ocupa, heurística se relacionará entonces con todo aquello que hace progresar una epistemología empirista; esto es, con todo lo que propenda al desarrollo de una epistemología basada en concepto de experiencia. Ahora bien, el hecho de que tanto semiótica como ciencia son, desde el punto de vista filosófico, filosofías empiristas, claramente permite imaginar que una pueda representar el rol de herramienta heurística-generador heurístico con respecto a la otra. En efecto, la semiótica puede generar nuevas formas de considerar problemas en epistemología científica y viceversa; pero nosotros en este artículo estamos interesados solamente en la primera situación. De manera que, en principio, lo que quiere decir que una podría representar un generador heurístico con respecto a la otra, básicamente, se trata de poder ver temáticas de una en términos de la otra. En otras palabras, la esfera semiótica puede mostrarse útil en generar nuevas perspectivas epistemológicas en filosofía de la ciencia, entendida esta como una epistemología empirista, tal y como fuera propuesto ya desde el propio título de este artículo. Siendo así, el potencial de la semiótica en tanto herramienta heurística en epistemología científica empirista es una posibilidad razonable. A continuación se discuten brevemente algunos ejemplos en donde, a nuestro entender, la semiótica ha desempeñado algún tipo de rol heurístico en episodios clásicos pertenecientes a la filosofía de la ciencia.

\section{TraZas Semióticas en algunaS REFERENCIAS CláSICAS DE LA FILOSOFÍA DE LA CIENCIA}

Proponemos en esta sección que la obra de algunos autores que representan referencias clásicas en filosofía de la ciencia presenta características semióticas, perceptibles sin raspar demasiado su superficie. Comenzamos con el caso de Rudolf Carnap, con obras como Empiricism, Semantics, Ontology (1974), y Philosophical Foundations of Physics, traducida al español como Fundamentación lógica de la física (1985). Con respecto a la primera referencia, el trasfondo semiótico debería saltar a la vista al ver propuesta una relación entre empirismo, semántica y ontología; efectivamente, la relación que propone Carnap 
se basa en que desde su punto de vista semántico, la espinosa cuestión de la referencia ontológica queda embebida en un contexto semiótico al trasladarla, desde la cuestión de la existencia de entidades abstractas, a la cuestión de la existencia de formas lingüísticas abstractas. Ahora bien, en cuanto a la segunda referencia, Carnap propone que entre los fundamentos filosóficos de la ciencia se encuentran dos elementos: los tres tipos de conceptos de la ciencia, que incluyen los conceptos cuantitativos, y el lenguaje que se construye a partir de ellos, el lenguaje cuantitativo; esto configura, por supuesto, otro contexto semiótico, localizado por Carnap a nivel básico en su obra que trata sobre los fundamentos filosóficos de la física (considérese la equívoca traducción del título de la obra referida).

Como ejemplos particulares de antecedentes semióticos, en la obra de Karl Popper es posible reseñar Conjeturas y refutaciones: el desarrollo del conocimiento científico (1983), y La lógica de la investigación científica (1995). En estas obras, Popper considera que la lógica de conjeturas y refutaciones de teorías científicas no solamente genera nuevas experiencias científicas, sino también proporciona los medios para integrarlas en las estructuras consistentes de los nuevos sistemas científicos (teorías científicas) producidos en el proceso. El trasfondo semiótico de estas obras se detecta claramente al caer en cuenta que una lógica de conjeturas y refutaciones de teorías científicas solamente es posible en el contexto (desarrollado incipientemente en ellas) de una lógica de la formación, evolución y cambio del contenido de los conceptos que permiten enunciarlas. En ese contexto eminentemente semiótico-, se hablaría de la dinámica de la subestructura semántica de los sistemas científicos (teorías científicas en este caso) en su calidad de sistemas semióticos; el contexto es semiótico porque una noción de los conceptos científicos así descrita los pone en el mismo estatus epistemológico que los conceptos indistintamente, no circunscrito solo a conceptos científicos-de un lenguaje. Por supuesto, lo relacionado con el lenguaje pertenece a la esfera semiótica por excelencia.

Del mismo modo, como ejemplo particular de trazas semióticas en la obra de Imre Lakatos pueden reseñarse Pruebas y refutaciones: la lógica del descubrimiento matemático (1986) y así La metodología de los programas de investigación científica (1993). Ahora es Lakatos quien considera, especialmente en la primera de estas obras, que la lógica de conjeturas y refutaciones de teorías matemáticas no solamente genera nuevas experiencias matemáticas, sino también proporciona los medios para integrarlas en las estructuras consistentes de los nuevos sistemas matemáticos (teorías matemáticas) producidos en el proceso. Puesto que el trabajo 
de Lakatos trata específicamente de teorías topológicas, las experiencias y estructuras generadas son entonces de carácter topológico. No es coincidencia que el trasfondo 'semiótico' de esta obra sea el mismo que el de la de Popper-Lakatos fue siempre un popperiano, nunca completamente convencido, sino persistentemente crítico-; de suerte que una lógica de pruebas y refutaciones de teorías matemáticas solamente es posible en el contexto (desarrollado incipientemente en la obra) de una lógica de la formación, evolución y cambio del contenido de los conceptos que permiten enunciarlas. Nuevamente, en ese contexto semiótico se hablaría de la dinámica de la subestructura semántica de los sistemas matemáticos (teorías matemáticas en este caso) en su calidad de sistemas semióticos.

Finalmente, como ejemplos particulares de trazas semióticas, en la obra de Paul Feyerabend pueden ser mencionados artículos como An attempt to a realistic interpretation of experience (1995a), On the interpretation of scientific theories (1995b), Explanation, reduction and empiricism (1995c), On the meaning of scientific terms (1995d), Reply to criticism: comments on Smart, Sellars and Putnam (1995e), Science without experience (1995 f), Linguistic arguments and scientific method (1995g) y Realism and instrumentalism, comments on the logic of factual support (1995h), entre otros. Estos títulos, y otros no referenciados aquí, dan claro indicio del trasfondo semiótico (inconscientemente desarrollado quizá) de su contenido, puesto que ubican conceptos semióticos en el centro de temáticas fundamentales en filosofía de la ciencia. Puede apreciarse la pertinencia de esta aseveración al notar lo siguiente, en su respectivo orden: el concepto de interpretación es una de las bases del tratamiento semiótico de la realidad ontológica, y aparece como protagónico ya desde el propio título de los artículos de Feyerabend (1995a y 1995b). Ahora bien, los conceptos de explicación y reducción que aparecen en el título de Feyerabend (1995c) son de vertiente semiótica, puesto que el alegato desarrollado en el artículo consiste en que una teoría física debe ser capaz de generar explicaciones de los fenómenos que estudia a través de descripciones reducibles a una hipotética entidad -semiótica- llamada lenguaje observacional, con tal de que este sea lo suficientemente claro en las relaciones que establezca para los contenidos de los conceptos observables. En cuanto al título de Feyerabend (1995d), resulta razonable inferir que una disertación a propósito del significado - meaning-de los términos científicos tenga connotaciones semióticas. Finalmente, en el título de Feyerabend (1995e) se hace referencia a una réplica del criticismo. El criticismo factualo fáctico al que responde Feyerabend se basa también en la mencionada hipotética existencia de la entidad semiótica llamada lenguaje observacional, que Feyerabend discute en detalle en el artículo. 
De esta manera, el visible tinte semiótico presente en las referencias anteriores permite sugerir que hacia el final de su carrera, Feyerabend se encontraba desarrollando una teoría del conocimiento científico de fundamentos semióticos que no alcanzó a proponer formalmente, pero sía esbozar preliminarmente en algunos de sus contenidos en obras como Tratado contra el método (1997), Adiós a la razón (1996), y Por qué no Platón (1993), así como en las conferencias Tridentinas, que sirvieron de base para el compendio temático editado con el título de Ambigüedad y armonía (1999a), así como también para la obra Conquest of abundance: a tale of abstraction versus the richness of being (1999b).

Ahora bien, debe recalcarse nuevamente que el desarrollo detallado de estas apreciaciones sobre el contenido del trabajo de los autores referidos no puede hacer parte del enfoque limitado de este artículo; sin embargo, a pesar de su generalidad, ellas permiten aseverar que un estudio en donde se desarrolle y argumente la posición de que parte de la obra de Popper, Lakatos y Feyerabend exhibe características semióticas es una posibilidad razonable. Asimismo, cumplen el papel de ilustrar genéricamente -si bien como hipótesis viables-, la utilidad, que ya no potencial, sino, en efecto, consumada, de la semiótica en tanto herramienta/ generador heurístico en filosofía de la ciencia.

\section{FUNDAMENTOS SEMIÓTICOS EN ALGUNOS ESTUdIOS EN FILOSOFÍA DE LA CIENCIA}

Por otra parte, a pesar de ser escasos, como se apuntó en los párrafos introductorios, existen, tanto a nivel latinoamericano como mundial, algunos estudios pertenecientes al contexto epistemológico de la filosofía de la ciencia, no relacionados con el concepto de experiencia, explícitamente apoyados en herramientas de tipo semiótico. Como ejemplo de latinoamericanos puede mencionarse la investigación desarrollada por Nancy Álvarez (Universidad de los Andes, Mérida-Venezuela), titulada La metáfora y el desarrollo científico, cuyo objetivo "fue analizar un concepto científico para explicitar su génesis metafórica, probando la suposición de que existe una génesis en las conceptuaciones científicas expresadas en niveles metafóricos de complejidad creciente" (1996, p. 17), teniendo como fundamento la "teoría de estructuración conceptual" (p. 16) desarrollada por Lakoff y Johnson en su obra Metaphors we live by (1980); y como ejemplo de los de fuera de Latinoamérica puede mencionarse la investigación desarrollada por René Thom(Institut des Hautes Études Scientifiques de Bures-sur-Yvette, Essonne-France), titulada Esbozo de una semiofísica: física Aristotélica y teoría de las catástrofes (1990), cuyo objetivo se refiere, en primer término, a la investigación de las formas significantes; apunta a 
construir una teoría general de la inteligibilidad (a partir de ellas como elementos fundamentales)... La hipótesis que presentamos aquí es la de que únicamente ciertas configuraciones de (estos) elementos tienen en verdad sentido y pueden servir de base a una construcción inteligible, susceptible de ser descrita lingüísticamente (mediante una ontología de raíces topológicas); se tratará de discernir, en el espectáculo ofrecido, elementos estables... que podrán obrar entre sí por contacto, fusionarse, escindirse, nacer y morir (desvanecerse) como seres vivos (Thom, 1990, p. 13-14).

\section{Discusión/CONCLUSIONES}

Esta última muestra de ejemplos permite apreciar que la utilización de herramientas semióticas en contextos de filosofía de la ciencia (incluyendo el de una epistemología empirista) es una tendencia en rápida expansión-si bien soterrada en muchos casos, quizá- de la investigación filosófica contemporánea. Desde el momento en que se publicaron las obras de los pioneros de la semiótica contemporánea hasta nuestros días no ha transcurrido un siglo, y desde su formalización en tanto disciplina académica no han transcurrido 50 años; a pesar de ello, la semiótica estructural-o mejor, la semiótica en general- se perfila como una infusión de renovación y dinamismo con respecto a disciplinas académicas más tradicionales. En primera instancia, ello podría significar que el decantado marco de referencia filosófico de la semiótica contemporánea muestra una promisoria capacidad para proporcionar herramientas con las cuales acceder a los más diversos campos de estudio; en otras palabras, ello significaría que, efectivamente, la semiótica ha podido y puede representar una suerte de "generador heurístico" en torno a diversas perspectivas propias de una epistemología empirista, relevantes en filosofía de la ciencia pasada y contemporánea; y en última instancia, ello podría significar tan solo el surgimiento y consunción de una moda pasajera. Sin embargo, debe observarse que si los autores de las investigaciones mencionadas en la sección inmediatamente anterior fuesen inquiridos por su parecer al respecto de todo este contexto, con toda seguridad encontrarían inaceptable la segunda opinión.

En conclusión, es razonable augurar un futuro prometedor a la semiótica en tanto herramienta de trabajo de naturaleza heurística en filosofía de la ciencia; probablemente, con el tiempo su accionar sea cada vez más explícito y diferenciadamente articulado. Ahora, en absoluto se nos antoja que resulte este un pronóstico intrépido; puesto que su pasado, aunque discreto y poco reconocido hasta elmomento, ha sido ya bastante significativo. 


\section{REFERENCIAS}

Álvarez, Nancy (1996). La metáfora y el desarrollo científico. Mérida: Consejo de Publicaciones de la Universidad de los Andes.

Carnap, R. (1974). "Empirismo, Semántica y Ontología”. (Empiricism, Semantics, Ontology). Trad. española de A. Deaño. En: Muguerza, J. La concepción analítica de la filosofía. Madrid: Alianza. Vol. II, p. 400-419.

Carnap, R. (1985). Fundamentación lógica de la física. Barcelona: Orbis.

Eco, H. (1994). La búsqueda de la lengua perfecta en la cultura europea. Barcelona: Crítica.

Feyerabend, P. (1993). Por qué no Platón. Madrid: Tecnos.

Feyerabend, P. (1995a). “An attempt to a realistic interpretation of experience”. En: Feyerabend, Paul. Philosophical papers. Cambridge: Cambridge University Press. Vol I, p. 17-37.

Feyerabend, P. (1995b). "On the interpretation of scientific theories”. En: Feyerabend, Paul. Philosophical papers. Cambridge: Cambridge University Press, Vol. I, p. 37-44.

Feyerabend, P. (1995c). "Explanation, reduction and empiricism". En: Feyerabend, Paul. Philosophical papers. Cambridge: Cambridge University Press, Vol. I, p. 44-97.

Feyerabend, P. (1995d). "On the meaning of scientific terms". En: Feyerabend, Paul. Philosophical papers. Cambridge: Cambridge University Press. Vol. I, p. 97-104.

Feyerabend, P. (1995e). 'Reply to criticism: comments on Smart, Sellars and Putnam”. En: Feyerabend, Paul. Philosophical papers. Cambridge: Cambridge University Press. Vol. I, p. 104-132.

Feyerabend, P. (1995f). "Science without experience". En: Feyerabend, Paul. Philosophical papers. Cambridge: Cambridge University Press. Vol. I, p. 132-137. 
Feyerabend, P. (1995g). "Linguistic arguments and scientific method". En: Feyerabend, Paul. Philosophical papers. Cambridge: Cambridge University Press, Vol. I, p. 146-161.

Feyerabend,P.(1995h). "Realism and instrumentalism: comments on the logic of factual support”. En: Feyerabend, Paul. Philosophical papers. Cambridge: Cambridge University Press, Vol. I, pp. 176-203.

Feyerabend, P. (1996). Adiós a la razón. Madrid: Tecnos.

Feyerabend, P. (1997). Tratado contra el método: esquema de una teoría anarquista del conocimiento. Madrid: Tecnos.

Feyerabend, P. (1999a). Ambigüedad y armonía. Barcelona: Paidós.

Feyerabend, P. (1999b). Conquest of abundance: a tale of abstraction versus the richness of being. Chicago: The University of Chicago Press.

Feynman, R. (1963). The Feynman lectures on physics. Volume I. Reading: AddinsonWesley Publishing Company, Inc.

Lakatos, I. (1986). Pruebas y refutaciones: la lógica del descubrimiento matemático. Madrid: Alianza.

Lakatos, I. (1993). La metodología de los programas de investigación científica. Madrid: Alianza.

Lakoff, G. and Johnson, M. (1980). Metaphors we live by. Chicago: The Chicago University Press.

Popper, K. (1983). Conjeturas y refutaciones: el desarrollo del conocimiento científico. Barcelona: Paidós.

Popper, K. (1995). La lógica de la investigación científica. Barcelona: Círculo de Lectores.

Rizzo Sierra, J.A. (2004). La metafísica de la experiencia. Trabajo de pregrado, UIS.

Thom, R. (1990). Esbozo de una semiofísica: física aristotélica y teoría de las catástrofes. Barcelona: Gedisa. 